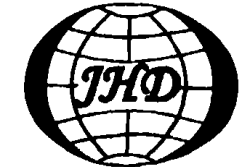

www.sciencedirect.com/ science/journal/10016058

\title{
COMBINATION OF CFD AND CSD PACKAGES FOR FLUID-STRUCTURE INTERACTION*
}

\author{
WANG Yi-wei \\ Institute of Mechanics, Chinese Academy of Sciences, Beijing 100190, China, \\ E-mail: wang_y_wei@yahoo.com.cn \\ LIN Yong-wen \\ China Academy of Space Technology, Beijing 100094, China
}

(Received October 9, 2007, Revised January 3, 2008)

\begin{abstract}
In this article the UDF script file in the Fluent software was rewritten as the "connecting file" for the Fluent and the ANSYS/ABAQUS in order that the joined file can be used to do aero-elastic computations. In this way the fluid field is computed by solving the Navier-Stokes equations and the structure movement is integrated by the dynamics directly. An analysis of the computed results shows that this coupled method designed for simulating aero-elastic systems is workable and can be used for the other fluid-structure interaction problems.
\end{abstract}

Key words: CFD/CSD, fluid-structure interaction, aero-elasticity fluttering

\section{Introduction}

Aero/hydro-elasticity problems arise in structures in air flows or water flows ${ }^{[1,2]}$. Such problems would be serious when the structure is thin, the stiffness is low and mutually coupled vibration is induced, which might cause structure damage. The aero-vehicle structure design looks for the light weight and it makes the fluttering problem more serious.

In the coupling system, the fluid and the solid obey their own equations ${ }^{[3]}$. Only the movement and stresses must be matched at their interfaces at any time. It is a very tough job for the airplane designer to derive and solve the equations which take the fluid part and the solid part as a whole. In order to solve the fluid-solid coupling problem, people used to assume tremendous simplifications, i.e., to assume the interface position is fixed, fluid is inviscous, etc. ${ }^{[4]}$

In recent decades, perfect commercial codes for fluid mechanics and solid mechanics are available which can solve very complicate engineering problems. The progress in CAD code development

Biography: WANG Yi-wei(1983-), Male, Master offers unified standard data form which is accepted by both fluid mechanics and solid mechanics. In such case, people can run the CFD and CSD code simultaneously and exchange numerical data on the interfaces in order to understand the coupling effect ${ }^{[5]}$.

Fluid-Structure Interaction ${ }^{[6]}$ (often referred to as FSI), is where fluid flow exerts pressure on a solid structure causing it to deform such that it perturbs the initial fluid flow. Aeroelastic analysis is one special kind of FSI problems, of which the interaction only performs on the interface of the fluid and structure, and there is remarkable relative motion. In aeroelastic instability, fluttering, which requires the most attention, usually results in catastrophic disaster to structures. One of the difficulties of the study on this phenomenon lies in its multi-patterns. In traditional methods, assumptions and approximations are usually applied. Now, however, due to the development of large-scale computers and the application of mature commercial software, it is possible to apply fewer or even no assumptions, which has been attempted in this work with the help of mature commercial software. In this article, a loosely-coupled Computational Aeroelastic Simulation (CAS) method is conducted, ${ }^{[7,8]}$ that is, the flow and the structure equations are 
treated separately, using synchronization procedure in space and time. More details of the method are described in the following sections

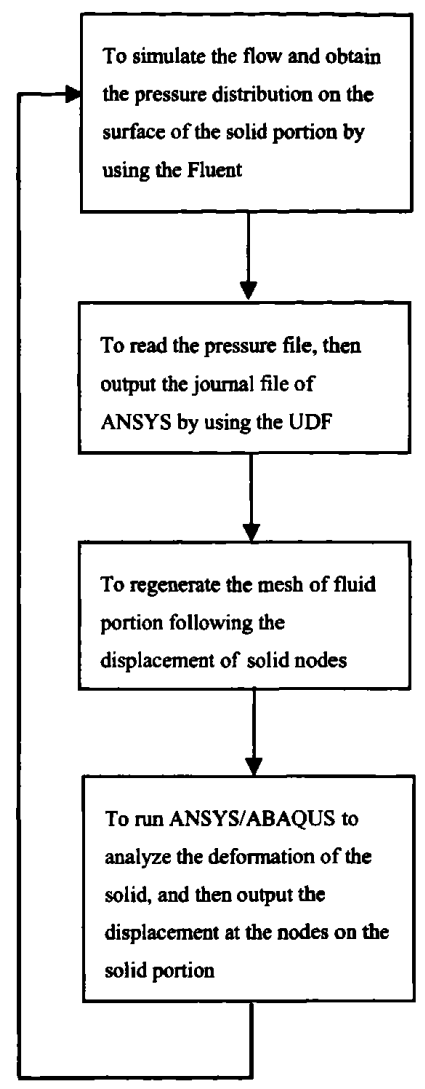

Fig.1 Process flow diagram

\section{Solution methods}

This loosely-coupled CAS method contains four main modules, and their functions are respectively (1) to simulate the flow and obtain the pressure distribution on the surface of the solid portion by using the Fluent, (2) to read the pressure file, then output the journal file of ANSYS by using the UDF, (3) to run the ANSYS/ABAQUS to analyze the deformation of the solid, and then output the displacement at the nodes on the solid portion, and (4) to regenerate the mesh of the fluid portion following the displacement of solid nodes by using the moving-mesh function of Fluent, and then go back to module 1. A flow diagram of the solution method is illustrated in Fig. $1^{[9]}$

\section{Applications}

3.1 3-D elastic flag swings in the wind

3.1.1 Problem description

This example presents a coupling simulation between ABAQUS and Fluent ${ }^{[10,1]}$. The elastic flag hangs on the top part of the channels. The angle between the normal of the flag and the normal of inlet is $20^{\circ}$. The velocity of the wind is $6 \mathrm{~m} / \mathrm{s}$. The scale of elastic flag is specified in Fig.2.

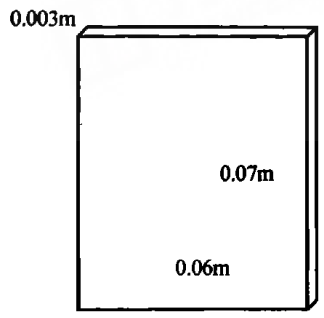

Fig.2 Shape of flag

\subsubsection{Solutions}

The mesh of Fluent is generated by ICEM and it is unstructured, which are illustrated in Figs. 3 and 4.

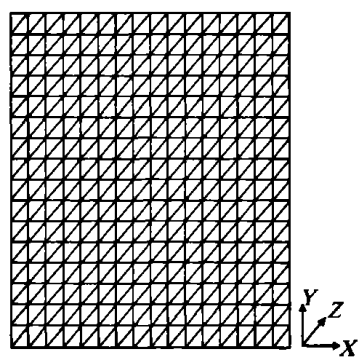

Fig.3 Mesh of flag used by Fluent

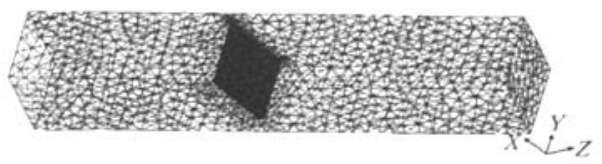

Fig.4 Boundary of fluid zone

In the Fluent portion ${ }^{[12,13]}$ the flag surface is specified as an adiabatic, moving, no-slip wall and the location of the nodes of flag surface is calculated by ABAQUS, and then updated by the Fluent dynamic mesh UDF. The front surface of channels is set as the velocity-inlet Boundary Condition $(\mathrm{BC})$, the back 
surface is set as the pressure-outlet BC, and the other surfaces of channels are set as walls. The situation is unsteady, and a segregated-implicit solver is used, with the $0.001 \mathrm{~s}$ per time step.

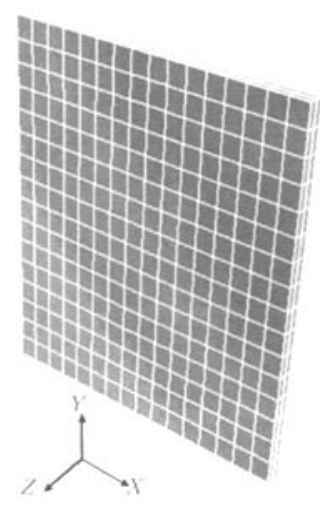

Fig.5 Flag mesh of ABAQUS

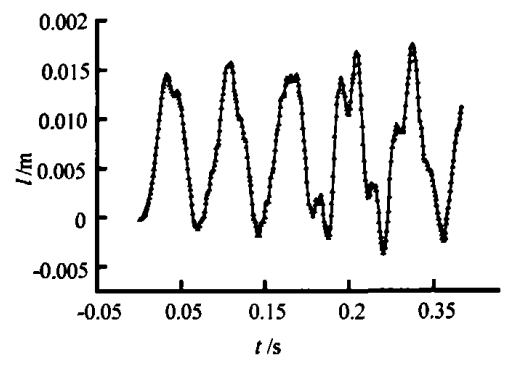

Fig.6 Curve for $z$ coordinates

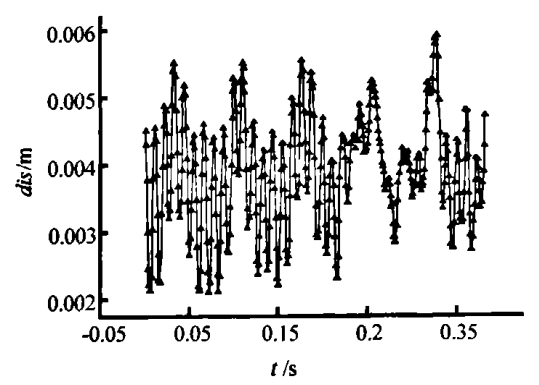

Fig.7 Curve for $y$ coordinates

In the ABAQUS portion ${ }^{[14]}$, all degrees of the upside of elastic flag are constrained. The nodes of surface mesh of ABAQUS have the same location as that of Fluent, as shown in Fig.5, and the element types are C3D8R. The ABAQUS solver is specified as explicit dynamic analysis, with geometry nonlinear switch opened. Every analysis step restarts from the result of pre-step. Surface node concentrated forces are obtained from the results of Fluent. The material property is constant, isotropic, and linear, Young's modulus is $2 \times 10^{6} \mathrm{~N} / \mathrm{m}^{2}$, Poisson's ratio is 0.45 , and the density is $3000 \mathrm{~kg} / \mathrm{m}^{3}$. The time step size is set as $0.001 \mathrm{~s}$, the same as that of the fluid portion.

\subsubsection{Results}

The whole simulation process uses $0.38 \mathrm{~s}$, the $z$ and $y$ coordinates of node $\left(\begin{array}{lll}-0.030 & 0.005 & 0.000\end{array}\right)$ changing with the time, are recorded (as shown in Figs.6 and 7). The torsion mode of flag is excited. The largest flag deformation is shown in Fig.8.
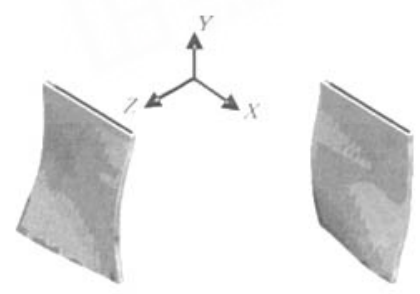

Fig.8 Largest deformation of flag

\section{3-D wing deformation in a subsonic flow \\ 4.1 Problem description}

This example $[15,16]$ involves the sweep-back wing shown in the Fig.9, the length of wing span is $1.5 \mathrm{~m}$, the root chord length is $1 \mathrm{~m}$ and the tip chord length is $0.6 \mathrm{~m}$. The wing has a leading-edge sweep angle of $40^{\circ}$ with the NACA0012 sections. All the degrees of freedom of the root nodes are constrained.

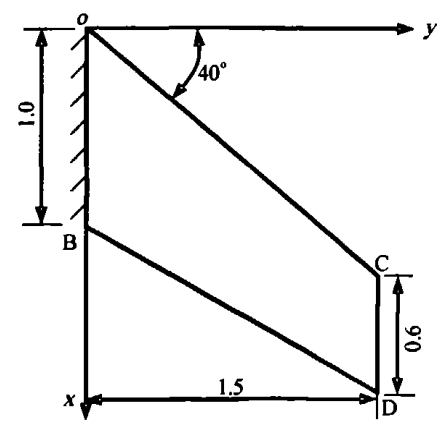

Fig.9 Shape of the wing

\subsection{Solutions}

. In this example, the unstructured mesh generated by ICEM is used, surrounded by a semi-cylinder boundary, and the grid near the wing is refined. There are about 150 thousand tetrahedral cells of the whole mesh and 1572 solid nodes on the wing surface, which are illustrated in Fig. 10. 


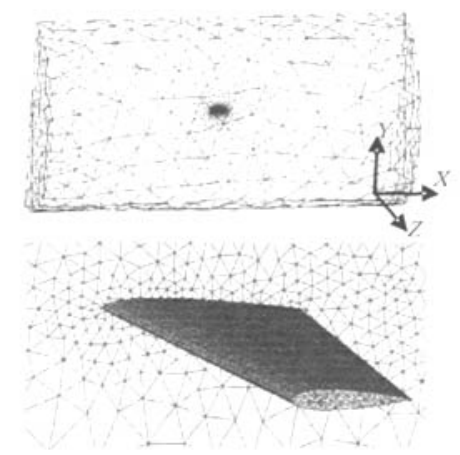

Fig.10 Mesh of the boundaries on the wing surface and symmetry

In the fluid portion ${ }^{[12,13]}$, the wing surface is specified as an adiabatic, moving, no-slip wall, and the velocities of the nodes are obtained from the result of ANSYS. The surface with the root of the wing is set as a symmetric one, and other outer surfaces surrounding the grid are set as the pressure far-field. The Mach number of the far field is $\mathbf{0 . 6}$, and the angle of attack is $10^{\circ}$. The unsteady, segregated-implicit solver is chosen as the numerical method, with a time step size of $0.002 \mathrm{~s}$.

The mesh for ANSYS is the same as the surface mesh of the wing in the fluid portion ${ }^{[14]}$, using the shell 63 elements, so there is no problem about interpolating. The solver of the solid portion is specified as a transient dynamic analysis using the full method. The material property is constant, isotropic, linear, Young's modulus is $12 \mathrm{GN} / \mathrm{m}^{2}$, Poisson's ratio is 0.3 , the density is $3000 \mathrm{~kg} / \mathrm{m}^{3}$ and the thickness is $0.05 \mathrm{~m}$. The time step size is set as $0.002 \mathrm{~s}$, the same as that for the fluid portion.

\subsection{Results}

With the time increasing, the wing vibrates in an approximate period, changing the flow, meanwhile, the lift and drag curves of the wing change, as shown in Figs. 11 and 12.

\subsection{Result analysis}

It can be seen that the lift and drag data are made up with several periodic elements of different frequencies, which are on the basis of wing's nature frequencies. For the purpose of confirming the result, a modal analysis is taken by the use of ANSYS, the result of which is shown in Table 1.

The foregoing six modes are illustrated in Fig.13. Considering that the lift coefficient (CL) and drag coefficient (CD) data reflect the holistic deformation of the wing, the Fast Fourier Transform (FFT) is taken to check the frequency components of the $\mathrm{CL}$ and $\mathrm{CD}$ data, and the frequency-amplitude obtained is shown in Figs. 14 and 15.

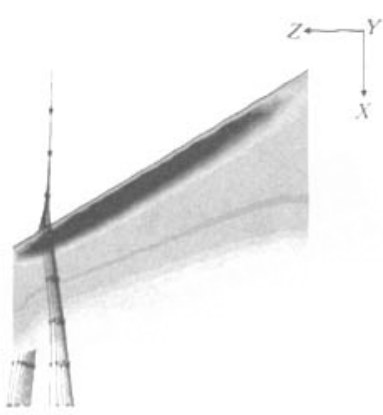

(a1) Flow stream over the wing

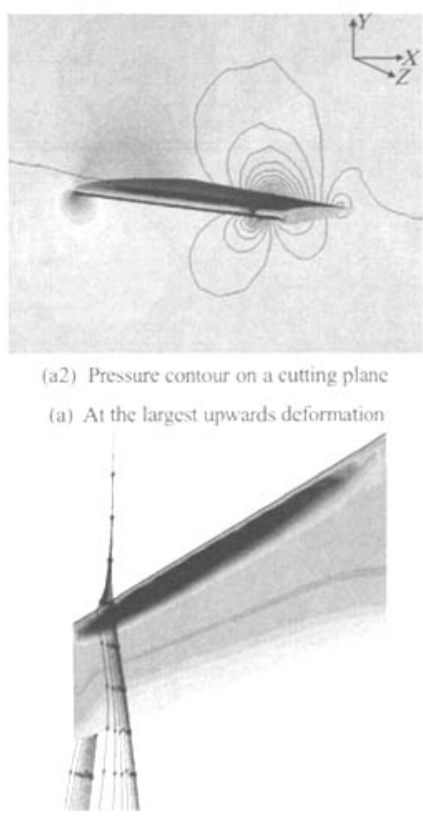

(b1) Flow stream over the wing

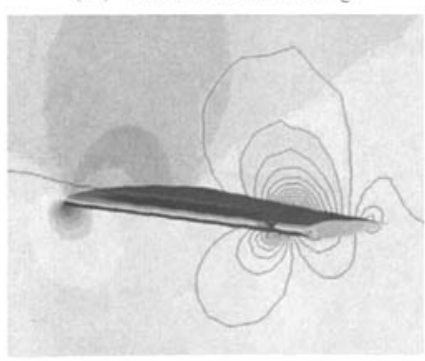

(b2) Pressure contour on a cutting planc

(b) At the largest upwards deformation

Fig.11 Flow streamlines and contours 

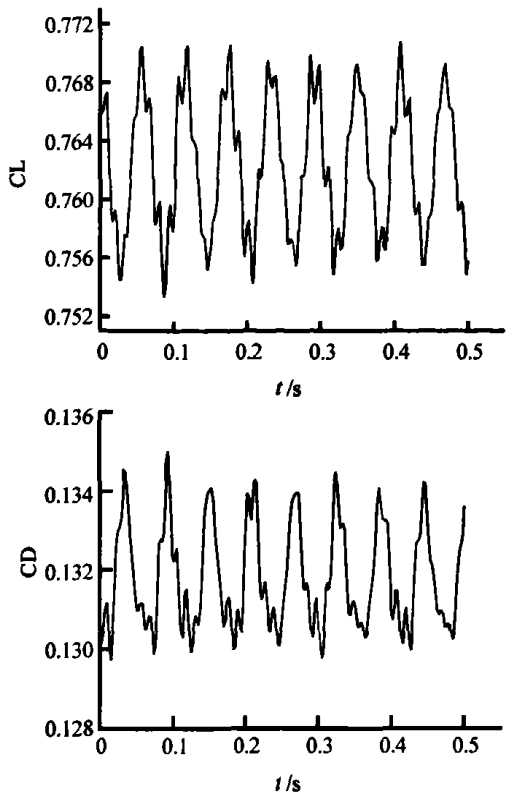

Fig.12 CL and CD curves
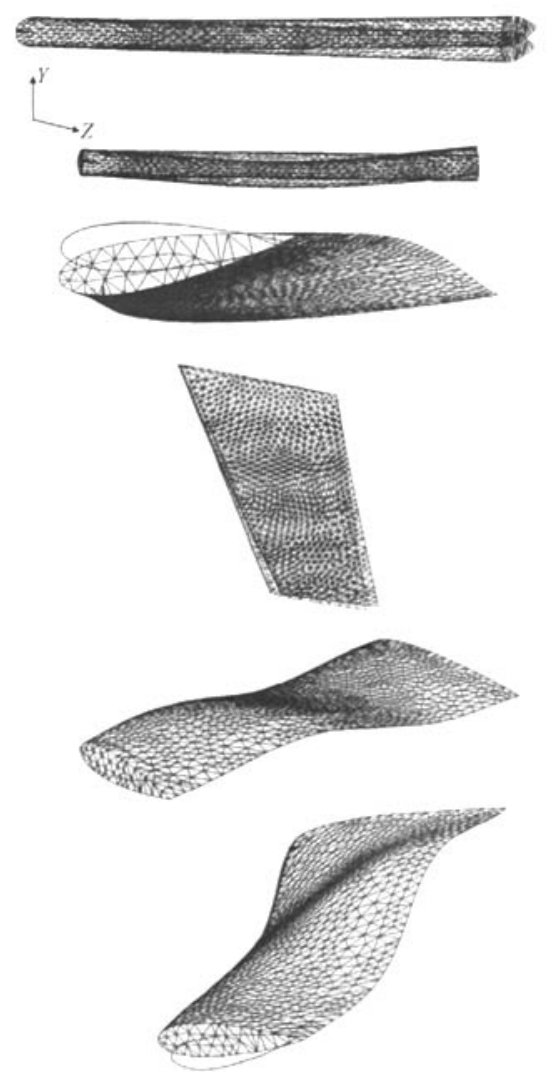

Fig.13 Mode shapes
Table 1 Results of modal analysis

\begin{tabular}{cccc} 
Mode & Frequency & Mode & Frequency \\
\hline 1 & 16.930 & 6 & 189.88 \\
2 & 72.999 & 7 & 191.92 \\
3 & 82.515 & 8 & 256.84 \\
4 & 87.951 & 9 & 268.47 \\
5 & 162.74 & 10 & 278.36 \\
\hline
\end{tabular}

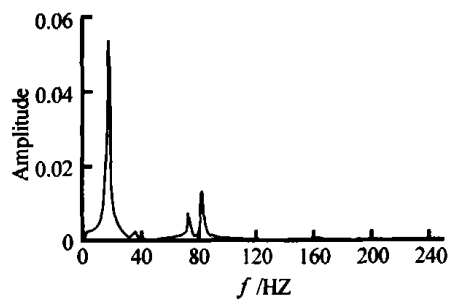

Fig.14 Curve for frequency-amplitude of $\mathrm{CL}$

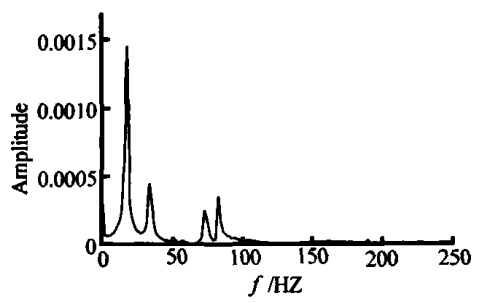

Fig.15 Curve for frequency-amplitude of CD

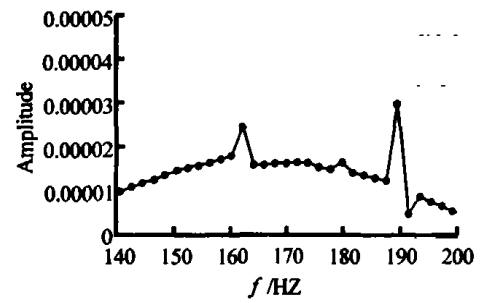

Fig.16 Partial enlargement of Fig.15

From the last figure, it can be seen that the peak frequencies correspond with the first order natural frequency, the octave of that, the second and the third order natural frequency. Figure 15 is zoomed in as Fig.16. 
The frequencies of those small peaks correspond with the fifth and sixth natural frequencies, but there is no peak corresponding with the fourth natural frequency, because the shape of the fourth order mode has little influence on the variety of the $C L$ and $C D$ data, which is shown in Fig.13.

\section{Conclusion}

This article provides an effective new idea to solve aeroelastic problem, in which the tools Fluent and ABAQUS/ANSYS employed are both effective and widely used $C F D / C S D$ commercial software. The results obtained also indicates that this coupling method is very accurate and logical, which can be used in engineering applications. Furthermore, this method is a general way that can also be employed to solve other kinds of fluid-structure interaction problems.

\section{References}

[1] DOWELL E. H., CURTISS H. C. and SCANLANET R. $H$. et al. A modern course in aeroelasticity[M]. Alphen aan den Rijn, The Netherlands: Sijthoff and Noordhoff International Publisher, 1978.

[2] ZWAAN R. J., PRANANTA B. B. Fluid/structure interaction in numerical aeroelastic simulation[J]. International Journal of Non-Linear Mechanics, 2002, 37: 987-1002.

[3] QUARTERONI A., VALli A. Numerical approximation of partial differential equations[M]. New York, USA: Springer-Verlag, 1998.

[4] LI Sheng-yuan., QIU Ji-bao. A treating method for coupling boundaries with large movement in fluid-solid interaction dynamics[J]. Journal of Astronautics, 2001, 22(1): 1-8(in Chinese).

[5] XU Min, AN Xiao-min and CHEN Shi-lu. CFD/ CSD
Coupling numerical computational methodology[ग]. Acta Aeronautica et Astronautica Sinica, 2006, 27(1): 33-37(in Chinese).

[6] XING Jing-tang., ZHOU Sheng and CUI Er-jie. A survey on the fluid-solid interaction mechanics[J]. Advances in Mechanics, 1997, 27(1): 19-38(in Chinese).

[7] GURUSWAMY G. P. Coupled finite difference/ finite element approach for wing body aeroelasticity[R]. AIAA Paper 92-4680, 1992.

[8] GURUSWAMY G. P., BYUN C. Fluid structural interactions using Navier-Stokes flow equations coupled with shell finite element structure[R], AIAA Paper 93-3087,1993.

[9] GURUSWAMY G. P. ENSAERO-A multidisciplinary program for fluid/structure interaction studies of aerospace vehicles[J]. Computing Systems in Engineering, 1990, 1( 2-4): 237-256.

[10] RELVAS A., SULEMAN A. Fluid-structure interaction modelling of nonlinear aeroelastic structures using the finite element corotational theory[J]. Journal of Fluids and Structures, 2006, 22(1): 59-75.

[11] GUO X., MEI C. Application of aeroelastic modes on nonlinear supersonic panel flutter at elevated temperatures[J]. Computers and Structures, 2006, 84(24-25): 1619-1628.

[12] ANDERSON J. D. Computational fluid dynamics: The basics with applications[M]. New York, USA McGraw-Hill, 1995

[13] PATANKAR S. V. Numerical heat transfer and fluid flow[M]. New York,USA: McGraw-Hill, 1980.

[14] WANG Xu-cheng. Finite element method[M]. Beijing: Tsinghua University Press, 2003 (in Chinese).

[15] HENSHAW M. J. de C., BADCOCK K. J. and VIO G. A. et al. Non-linear aeroelastic prediction for aircraft applications[J]. Progress in Aerospace Sciences, 2007, 43(4-6): 65-137.

[16] RAO V. M., BEHAL A. and MARZOCCA P. et al. Adaptive aeroelastic vibration suppression of a supersonic airfoil with flap[J]. Aerospace Science and Technology, 2006, 10(4): 309-315. 